\title{
L'OUvroir de PAYsage POtentiel
}

\author{
Danièle Méaux ${ }^{1}$
}

À partir des années soixante, certains auteurs ont théorisé la valeur productive des règles arbitraires. Nombre d'artistes contemporains ont également eu recours à la contrainte ${ }^{2}$. Mais celle-ci attire également ceux (hommes de lettres, photographes ou artistes...) qui font du voyage la matière même de leur œuvre. Dès lors, la contrainte se fait levier d'une investigation renouvelée des sites; elle trouve en la photographie un allié privilégié.

Les déplacements devenant plus faciles et les représentations du lointain plus accessibles, le vingtième siècle est marqué par un reflux de la sensation d'exotisme ; nombreux sont les chants de deuil, chez les écrivains voyageurs de notre temps : Victor Segalen, Claude LéviStrauss, Paul Morand déplorent l'uniformisation du monde et le déclin du véritable voyage d'exploration. De façon concomitante, l'attention tend à se déporter vers des territoires proches ou sous-évalués, dont la

1 IUFM Amiens et CIEREC - Université de Saint-Étienne.

2 Voir à cet égard, Z. AYDIN, Émergence de la notion de contrainte en art, thèse de doctorat en Arts Plastiques soutenue à Paris VIII, sous la direction de Laura Malvano, 2002.

Recherches en communication, $\mathrm{n}^{\circ} 27$ (2007). 
perception peut être renouvelée. Certains ethnologues se tournent vers des enquêtes de proximité : Marc Augé réalise ainsi un travail d'observation dans le métro parisien ${ }^{1}$. Des écrivains (Jean Rolin, François Bon, Paul Théroux, Georges Picard...) effectuent des trajets dans des régions voisines et banales. Des photographes (Thierry Girard, Claude Pauquet, Bernard Plossu...) portent leur attention sur des territoires proches qui ne «valent pas le détour »- pour reprendre une expression employée dans les guides verts. Georges Perec note : «[D]ans l'immeuble où l'on habite [...] quelque chose qui peut ressembler à du dépaysement peut venir du fait que l'on prendra l'escalier B au lieu de l'escalier A, ou que l'on montera au $5^{\mathrm{e}}$ alors que l'on habite au second $»^{2}$. La qualité de perception que l'on impute ordinairement au voyage n'est pas dépendante de la distance parcourue, ni de l'originalité des sites ; elle tient au regard que l'on porte sur les lieux, qui doit être décapé du sommeil de la routine.

Mais renouveler l'attention que l'on pose sur des sites « gris » n'est pas une entreprise aisée. Les habitudes s'avèrent souvent pesantes et anesthésiantes. L'élection d'une contrainte se présente dès lors comme un levier qui peut permettre de percevoir les choses autrement. Pour le déplacement (comme pour le texte littéraire), l'adoption d'une règle est générative de renouvellement. Que l'on écrive ou que l'on voyage, la liberté apparaît comme un leurre ${ }^{3}$ : l'être (itinérant ou parlant) est soumis à des pressions et à des habitudes. Choisir une contrainte, c'est être conscient de ces déterminations et vouloir les perturber.

\section{Contraintes géographiques}

Décider de suivre un axe déterminé par certaines configurations géographiques peut constituer un premier type de contrainte de déplacement. Alors que, généralement, on voyage pour aller quelque part, certains peuvent décider de suivre une autoroute, une nationale, une frontière ou un fleuve. Ils respectent dès lors un itinéraire imposé par la conformation du territoire et explorent les possibilités qu'il offre. En optant pour la soumission à une règle, le voyageur manifeste la différence entre son voyage et un « transport» ordinaire. L'existence

1 M. AUGÉ, Un ethnologue dans le métro, Paris, Hachette, « Littératures », 1986.

2 G. PEREC, Espèces d'espaces, Paris, Galilée, «L'espace critique », 1974, p. 62.

3 H. LE TELliER, Esthétique de l'Oulipo, Paris, Le Castor Astral, 2006. 
d'un contrat préétabli fait du sujet itinérant le décideur conscient de son voyage, et en même temps le prive, à partir d'un certain moment, de toute initiative de sorte qu'il reçoit dès lors chaque perception comme une chose qui lui est imposée. Il est de nombreux exemples de Voyage de ce genre : Bernard Plossu emprunte la Route nationale $1^{1}$, Christian Louis, la Nationale $7^{2}$, Thibaut Cuisset, la Route de Paris à Montreuil ${ }^{3}$; Thierry Girard suit le Danube, le Rhin, la frontière belge, Claude Pauquet longe le littoral de Dunkerque à Hendaye ${ }^{4}$, Paul Théroux fait le tour de la Grande Bretagne en chemin de fer ${ }^{5}$, etc.

\section{Contraintes culturelles}

L'itinéraire élu peut aussi être celui qu'a emprunté précédemment un autre voyageur. Dans La Route de Tôkaidô, Thierry Girard part sur les traces de Hiroshige ${ }^{6}$. Didier Sorbé fait l'ascension du Mont Perdu selon l'itinéraire de Louis Ramond de Carbonnières (1802)7 . Philippe Séclier va de Vintimille jusqu'à Trieste, par la route qu'a empruntée Pier Paolo Pasolini ${ }^{8}$; Françoise Huguier refait le chemin de Michel Leiris en Afrique $^{9}$, Gilles Mora, celui de Walker Evans dans le Deep South ${ }^{10}$. Ces entreprises sont des hommages, des pèlerinages. Mais ce sont aussi des « voyages à contrainte ». La règle choisie n'est pas arbitraire ; elle est au contraire motivée par certaines affinités ou par l'admiration portée à une œuvre. Mais chaque voyage relaté constitue un programme de

1 B. Plossu, Route Nationale 1, Douchy-les-Mines, Centre Régional de la Photographie Nord-Pas-de-Calais, Mission Photographique Transmanche, Cahier 10, 1992.

2 C. LOUIS, Nationale 7, Paris, Marval, 1988.

3 T. CUISSET, La rue de Paris à Montreuil, Trézélan, Filigranes Éditions, 2005.

4 C. PAUQUET, Bordures. Un parcours de Dunkerque à Hendaye, Dunkerque Poitiers, MJC Rosendaël / Maison des trois quartiers, 1995.

5 P. THÉROUX, Voyage excentrique et ferroviaire autour du Royaume-Uni [1983], Paris, Grasset, « Les Cahiers rouges », 1993. Le titre original est The Kingdom by the sea.

6 T. GIRARD, La route de Tokaidô, Paris, Marval, 1999.

7 D. SORBÉ, R. DAY, Mont-Perdu, Pau, Éditions du Pin à Crochets, 2003.

8 P. SÉCLIER, Pier Paolo Pasolini. La longue route du sable, Paris, Éditions Xavier Barral, 2005.

9 F. Huguier, M. CRESSOlE, Sur les traces de l'Afrique, Paris, Maeght Éditeur, 1990.

10 G. MORA, C. NORI, L'été dernier - Manifeste photobiographique, Paris, Éditions de l'Étoile, «Écrit sur l'image », 1983. 
déplacement, qui n'appartient pas en propre à son auteur, mais peut être successivement décliné par des voyageurs ultérieurs, comme un poème oral est repris par tel ou tel aède, sans que l'on puisse parler de plagiat ou de copie.

\section{Contraintes arbitraires}

Il est enfin des contraintes de déplacement qui ont un caractère délibérément arbitraire. On pense au Testament d'un excentrique de Jules Verne où les personnages parcourent les États-Unis en respectant un ordre alphabétique, à Mobile de Michel Butor où le passage d'un État à un autre tient à l'homonymie de certains noms de ville. Il s'agit là d'itinéraires lointains. Mais l'adoption d'une contrainte paraît singulièrement efficace dans le cas de « voyages de proximité »; le recours à un artifice permet alors le renouvellement de l'attention.

Dans Les Passagers du Roissy-Express, François Maspero et la photographe Anaïk Frantz empruntent le RER B de Roissy à SaintRémy-lès-Chevreuse, en s'arrêtant pour dormir à chaque station (excepté quand ils sont à l'intérieur de Paris). La contrainte de devoir, chaque soir, trouver un hébergement les met en situation de voyage ; elle les amène aussi constamment à prendre les lieux à rebours de leur utilisation ordinaire, puisqu'il leur faut raccorder des endroits relativement proches à vol d'oiseau, mais que l'on n'a pas coutume de relier.

Pour gagner à pied le Relais bleu, il faut passer sous l'autoroute, à l'endroit même où les deux branches venues l'une de la porte d'Italie et l'autre de la porte d'Orléans se rejoignent. C'est un passage fait de rampes, d'escaliers et de tunnels déserts où stagnent des flaques d'urine, ponctué de sculptures abstraites faites de ciment et de pots cassés dans des sortes de jardinets de cailloux [...]. ${ }^{1}$

L'entreprise des deux auteurs permet de faire apparaître certaines aberrations en matière d'aménagement du territoire. En écho à la relation de François Maspero, certaines photographies d'Anaïk Frantz montrent

1 F. MASPERO, A. FRANTZ, Les passagers du Roissy-Express, Paris, Seuil, « Fiction \& Cie », 1990, p. 274. 
des espaces laissés en friche, entre échangeurs et rocades ${ }^{1}$. Mais les vues très sobres présentent surtout des êtres humains, qui sont autant de rencontres de voyage. Le texte effectue de constants rappels historiques sur les lieux traversés : il retrace, par exemple, toute l'histoire de la cité des 3000, près d'Aulnay-sous-Bois ; il remonte à l'époque où l'aéroport Charles de Gaulle n'existait pas et où Roissy était un village. Aux espaces déshérités de la banlieue, l'écrivain restitue une histoire. Mais cette perception renouvelée de territoires ordinaires n'est rendue possible que grâce au contrat qui a nettement été posé au départ :

[...] il avait consulté le plan du métro régional express et il avait vu que la ligne comptait 38 gares, y compris celles de la traversée souterraine de Paris, pour un parcours d'environ 60 kilomètres, et qu'à raison d'une gare par jour, en sautant celles de Paris et en en gommant quelques petites, ce serait près d'un mois qui lui serait nécessaire pour mener à bien un tel voyage. Un mois au cours duquel, avait-il décidé, il ne rentrerait jamais à Paris.

[...] Ce serait un vrai voyage : chaque soir, il faudrait chercher un hôtel. Ou alors s'arrêter chez des amis. ${ }^{2}$

Sur cette « règle du jeu », l'écrivain revient à plusieurs reprises. Dans les faits, les deux voyageurs sont amenés à faire une ou deux entorses au protocole, quand ils ne trouvent vraiment pas où dormir ; mais cela reste exceptionnel. La contrainte de départ est essentielle à l'entreprise. Elle régit l'expérience, conditionne le commerce avec les hommes et avec le territoire.

Les Autonautes de la cosmoroute ${ }^{3}$ est le récit à deux voix d'un voyage de Paris à Marseille : les deux compagnons se sont donné pour loi de s'arrêter sur toutes les aires d'autoroute, et de passer la nuit toutes les deux aires, dans leur camionnette Volkswagen surnommée « Fafner ». Il y a soixante-dix aires entre Paris et Marseille; le parcours dure donc plus d'un mois. Carol Dunlop et Julio Cortázar se font ravitailler en produits frais par des comparses. Une extrême importance est accordée à la notion même de règle :

1 Ibid., pp. 46, 191...

2 Ibid., p. 15.

3 C. DUNLOP, J. CORTÁZAR, Les autonautes de la cosmoroute ou un voyage intemporel Paris-Marseille, Paris, Gallimard, « NRF », 1983. 
Je me souviens des règles de la marelle, des billes, de la toupie et de l'accès progressif à d'autres règles qui peu à peu m'enfermaient dans le monde des adultes : le ludo, les dames, les échecs : pièces touchée-pièce jouée, souffler n'est pas jouer, tout minutieusement statué, inévitable et parfait, comme deux et deux font quatre ou les campagnes militaires du général San Martin. Ainsi, aujourd'hui et les trente-deux aujourd'hui qui restent : on-ne-sort-pas-de-l'autoroute. ${ }^{1}$

Carol Dunlop et Julio Cortázar s'amusent constamment de la délicieuse possibilité d'une transgression du protocole établi. Les deux auteurs paraissent pris dans la jubilation du respect de cette règle qu'ils se sont fixée pour un mois. Elle manifeste leur connivence, les installe dans un univers privé qui diffère de celui des autres. C'est un espace de jeu, n'appartenant qu'à eux, que les deux amants se sont savamment circonscrit.

L'entreprise de Carol Dunlop et Julio Cortázar - comme celle de François Maspero et Anaïk Frantz - tient un peu de la performance ; l'expérience vécue a la plus grande importance. Le livre mêle textes, photographies et dessins, en une mise en page un peu brouillonne. Les vues prises sur le vif paraissent répondre au seul désir de consigner le vécu. Elles présentent l'un ou l'autre des deux voyageurs, leur camionnette, les aires d'autoroute et leurs installations (WC, poubelles, plantations...). Le texte, quant à lui, est très composite, croisant des écrits de chacun des deux auteurs (dont les tonalités diffèrent) avec des fragments de « carnet de bord» ou des lettres soi-disant trouvées. Le protocole établi permet d'utiliser l'autoroute à contre-emploi : un monde de la rapidité se trouve transformé en monde de la lenteur. Pour l'automobiliste, la vitesse fait du territoire un spectacle irréel, défilant autour de l'habitacle clos ; elle a un effet dématérialisant ${ }^{2}$. Par leur allure d'escargots (comme ces derniers d'ailleurs ils voyagent accompagnés de leur coquille «Fafner »), Carol Dunlop et Julio Cortázar se situent aux antipodes de cette perception du territoire ; ils constatent «l'altération insensible de la notion habituelle d'autoroute »; « sa fonctionnalité insipide et presque abstraite fait place à une présence pleine de vie et

1 Ibid., p. 47.

2 P. VIRILIO, Esthétique de la disparition [1979], Paris, Le livre de poche, « Biblio essais », 1994, p. 77. 
de richesse : les gens, les haltes, les épisodes vécus $[\ldots] »^{1}$. Les lieux d'étape se trouvent pour ainsi dire « re-matérialisés »; ils sont le théâtre d'interactions entre les personnes qui s'y arrêtent ou y travaillent. Chaque aire s'avère différente de la voisine :

Les parkings sont ce qu'ils sont, pauvres anges, mais chacun représente une modification intéressante à l'intérieur d'un schéma unique. [...] nous découvrons chaque fois une légère altération dans la place des éléments habituels. Les WC par exemple que nous avions à dix mètres à droite se trouvent cette fois à vingt mètres à gauche, ou derrière nous ; le petit bois aux troncs sveltes de cette nuit est devenu une masse de chênes imposants d'où tombent de sympathiques petits vers [...] les tables avec leurs bancs de grosses planches où il est agréable de déjeuner ou de dîner se distribuent différemment ainsi que les boîtes à ordures dont la généreuse abondance nous comble. ${ }^{2}$

C'est une « autoroute parallèle $»^{3}$ que la contrainte permet aux deux compagnons de connaître.

Les déplacements relatés par François Bon dans Paysage fer sont entrepris pour raisons professionnelles ${ }^{4}$. Mais afin de transformer l'expérience, l'écrivain se dote d'une règle qui est tout à la fois contrainte d'écriture et protocole d'observation des territoires traversés - l'une et l'autre étant intrinsèquement liés ; il décide de consigner à chaque trajet ce qu'il voit, mais sans jamais rien soustraire à ce qui a déjà été écrit, en ajoutant toujours des lignes supplémentaires. Chaque déplacement entraîne un allongement du texte et les mêmes sites se trouvent nouvellement appréhendés. Parfois des observations identiques sont répétées. Mais fréquemment aussi de nouveaux éléments, des détails supplémentaires sont perçus. Le retour du même permet à l'auteur de se forger des repères, à partir desquels s'opère un élargissement, par touches successives, de la perception du territoire. La discipline que l'auteur s'est fixée présente une homologie évidente avec la contrainte instaurée par le déplacement ferroviaire, qui ne laisse pas au passager la possibi-

1 C. DUNLOP, J. CORTÁZAR, op. cit., pp. 67-68.

2 Ibid., p. 81.

3 Ibid., p. 40.

4 Il s'agit pour l'écrivain parisien d'aller chaque semaine à Nancy où il anime un atelier d'écriture. 
lité de s'arrêter ou de revenir en arrière. Mais François Bon choisit de ne pas subir cette privation de liberté, en la mettant en quelque sorte dans son propre jeu. L'intérêt de l'auteur se porte sur la progressive construction de la perception. Au fil des ajouts successifs dans la linéarité du texte, les paysages ordinaires et gris, émaillés de parkings, de surfaces commerciales, de friches industrielles... prennent corps ; ils s'enrichissent de détails et de qualités sensibles. Des traits anodins se font les indices de modes de vie que l'écrivain s'essaie à reconstituer ; des indices renvoient à une activité industrielle qui désormais n'est plus et signent certaines difficultés économiques.

Il est aussi maints photographes voyageurs qui optent pour une contrainte délibérément arbitraire. En 1985, dans le Pas-de-Calais, Pierre Devin trace une ligne entre Fourmies et Cap Gris-Nez qu'il s'astreint à suivre ; l'exposition réalisée ensuite s'intitule Diagonale; le titre attire l'attention du visiteur sur la démarche du praticien. Thierry Girard déclare : «Mon travail s'est depuis longtemps constitué autour de l'idée d'un parcours contraint par un itinéraire plus ou moins élaboré [... $»^{1}$. Pour D'une mer l'autre ${ }^{2}$ (2002), une droite tracée sur une carte détermine son déplacement, de Nice à Ouessant. Sur la couverture du livre, une photographie en couleur montre des sables et des traînées d'eau ; l'image est fendue d'une puissante oblique descendante, qui possède la forme contournée et irrégulière des routes sur les cartes et la couleur rouge généralement impartie aux nationales. Pour la réalisation de Voyage en Saintonge (1995), c'est l'enroulement d'une spirale qui commande ses pas : " J'avais tracé sur ma carte, grossièrement, une sorte de spirale pour que le chemin qui me mène jusqu'à Saintes soit le plus long possible et ne recoupe jamais un lieu déjà traversé. $»^{3}$.

1 T. GIRARD, "La route de Tôkaidô", Une route, un fleuve. Itinéraires récents au Japon et en Europe Centrale, catalogue de l'exposition organisée à la Galerie de la Marine à Nice du 5 décembre 1998 au 24 janvier 1999, Nice, Éditions de la ville de Nice, 1999, ouvrage non paginé. C'est moi qui souligne.

2 T. GIRARD, D'une mer l'autre, Paris, Marval, 2002.

3 T. GIRARD, Un voyage en Saintonge, Saintes, Ccr de L'Abbaye aux Dames, 1995, ouvrage non paginé. « Cagouille » est un régionalisme pour « escargot». 


\section{Un OUvroir de PAYsage POtentiel}

S'imposer des règles arbitraires permet de sortir des sentiers battus : se fixer des lois de type idiosynchrasique aide à s'écarter de la loi respectée par le plus grand nombre et instituée par la culture. La démarche, qui déroge aux usages, présente une allure de désobéissance. Parmi les auteurs voyageurs contemporains (qu'ils soient écrivains ou photographes), le recours à de telles contraintes est fréquent ; un protocole clairement revendiqué régit la traversée des territoires. La procédure élue touche en premier lieu l'appréhension du réel, et non la façon dont on en rend compte (même si bien sûr elle rejaillit sur cette dernière) ; touchant tout d'abord (si je puis dire) le voyage, elle concerne donc de façon comparable hommes de lettres et hommes d'images. Elle n'est pas tendue vers une finalité formelle, mais plutôt vers la découverte du réel qu'elle modifie (même si elle rétroagit évidemment sur la transcription de l'expérience). Dans Perec/rinations, Georges Perec propose de la sorte des parcours tautogrammatiques - les noms de rue doivent commencer par la même lettre -, monothématiques - rues à noms d'oiseaux, de villes, de musiciens... - ou encore alphabétiques allant de A jusqu'à $\mathrm{Z}$ - conditionnant une nouvelle appréhension de la ville ${ }^{1}$. Il offre au promeneur des procédures pour enrichir le répertoire de ses itinéraires, comme on étoffe les tournures de son langage. La perception de la ville y gagne en diversité, en richesse.

De tels travaux (de photographes ou d'écrivains) ont une composante expérimentale; les protocoles établis permettent de pousser le voyageur hors de ses habitudes, le conduisent vers de nouvelles relations du territoire ; elles semblent capables de restaurer l'aventure, le goût pour la vie et le monde. Le choix de ces contraintes travaille à une forme d'OUvroir de PAYsage POtentiel. Elles constituent des stratégies pour révéler les potentialités du territoire et manifestent une forme de foi dans les ressources des sites. Dans le même ordre d'idée, existent un OUvroir de CARtographie POtentielle, un OUvroir de POlitique POtentielle, un OUvroir de JArdinage POtentiel, un OUvroir de CUIsine POtentielle, un Ouvroir de GÉOgraphie POtentielle...

1 G. PEREC, Perec/rinations [1980-1981], Cadeilhan, Zulma, 1997. 
Le « Paysage » n'est pas le «Pays », la « contrée » effectivement visitée, mais davantage le rapport complexe, fait de sensations diverses et réparties dans la durée, de souvenirs et des savoirs aussi, qui s'instaure entre le sujet et le territoire. Il est évidemment empreint de culture. Les théories contemporaines font du " paysage » un espace de " médiance », un lieu d'échange entre l'homme et l'environnement ${ }^{1}$. Il ne s'agit donc pas seulement de dire que le choix d'un itinéraire contraint amène à passer par des endroits qui sont ordinairement ignorés (même si cela est parfois vrai) mais plutôt d'affirmer qu'il instaure une autre relation globale au pays traversé, les habitudes étant prises à rebours, les hiérarchies et les perceptions étant redistribuées, l'attention aiguisée, les rythmes modifiés... Parler d'OUvroir de PAYsage POtentiel, c'est prendre en compte le fait que le paysage est lié à des pratiques qui permettent au sujet que s'instaure un échange entre intériorité et extériorité. Le « pays » porte la marque des activités humaines ; mais le « paysage »- en tant que relation - est conditionnée par les conduites : les contraintes permettent de rompre avec la répétition et de travailler à l'instauration de nouveaux échanges avec les sites.

Pour les oulipiens, le choix de la contrainte, revient à supposer qu'il n'y a pas de matériaux ou de sujets mineurs ; tout peut-être pris en considération. L'élection d'une règle arbitraire accompagne un intérêt pour l'ordinaire ou la méconnu. Plus que la représentation du banal, c'est sa perception que la contrainte permet ici peu ou prou de forcer. Les règles que s'imposent François Maspero et Anaïk Frantz, Carol Dunlop et Julio Cortázar, Thierry Girard, perturbent les habitudes de déplacement et de perception, modifient les allures. Elles instaurent un écart productif. Elles placent le voyageur dans une posture qui induit la surprise. Jorge Luis Borges écrit : « Il n'y a qu'un cas où une œuvre ne vaut rien : c'est quand elle correspond aux intentions de l'auteur. $»^{2}$. On pourrait également dire que le déplacement vaut davantage quand il ne suit pas les pulsions instinctives du voyageur. Contraint, le parcours permet de libérer les potentialités du paysage, même le plus ordinaire.

Les situationnistes cherchaient également à réveiller l'appréhension du tissu urbain, en contrecarrant les habitudes ; leur approche laissait

1 A. BERQUE, Les raisons du paysage, Paris, Hazan, 1995.

2 J. L. BORGES, E. SABATO, Conversations à Buenos Aires, Éditions du Rocher, 2001, p. 35. 
une place à l'aléatoire. Dans le sillage du situationnisme, certains artistes contemporains de la déambulation urbaine œuvrent à une réinvention de la perception de la ville. C'est par exemple le cas du « Laboratoire d'Art Urbain Stalker $»^{1}$; depuis 1996, ce collectif a effectué de longs parcours dans les banlieues et les zones laissées en friche, qui se situent à la marge des grandes agglomérations ${ }^{2}$. Ces déplacements supposent des contraintes d'itinéraire. À Rome, les marcheurs sont partis d'une gare désaffectée, ont traversé les champs, les voies ferrées ; ils ont campé sur un terrain de foot, dans le chantier de construction d'une rocade... Les membres du collectif s'intéressent aux «vides » urbains qui sont en perpétuelle transformation et dont le devenir n'est pas planifié ; ces zones constituent, à leurs yeux, des endroits où se joue l'avenir des agglomérations ; ce sont, pour eux, les «combles de la ville $»^{3}$. Leurs marches sont souvent consignées par des prises de vues ou des enregistrements vidéo. "Nous avons choisi le parcours comme la forme d'art qui permet de souligner un lien en traçant physiquement une ligne $[\ldots] \gg^{4}$ : leurs déplacements - qui supposent un protocole - débouchent sur une réévaluation des sites, sur la réactivation du regard porté sur les lieux.

Le « PILI » (Plan Indicateur Lumineux d'Itinéraires) se situe quant à lui dans une plus stricte filiation oulipienne. Il fut réalisé par l'artiste Philippe Favier en collaboration avec Jacques Roubaud, à l'occasion du centenaire du métro de Paris. Sur un plan lumineux présenté à la station «Pyramides » (ligne 14), 74 itinéraires contraints sont proposés aux voyageurs (trajets lipogrammatiques, trajets d'écrivains, trajets alphabétiques... $)^{5}$. Le soin d'identifier les contraintes est laissé aux voyageurs. Ceux qui le souhaitent peuvent évidemment expérimenter les itinéraires proposés. Il est là encore question de perturber la perception assoupie de la ville.

1 Ce nom « Stalker » vient d'un film homonyme d'Andreï Tarkovsky (1979), dans lequel un passeur (stalker) conduit deux personnes d'une ville vers une zone interdite ; ils partent en quête d'une chambre dans laquelle tous les désirs peuvent se réaliser ; mais c'est surtout l'errance que connaissent les deux hommes.

2 Voir, à cet égard, T. DAVILA, Marcher, créer. Déplacements, flâneries, dérives dans l'art de la fin du XX $X^{e}$ siècle, Paris, Éditions du Regard, 2002, pp. 119-120.

3 Voir www.stalkerlab.it

4 Ibid.

5 Voir à ce sujet L. BOUDIER, PILI, une œuvre de Philippe Favier. Parcours de Jacques Roubaud, Paris, Le Flohic / RATP, 2001. 


\section{Contraintes et photographie}

Toutes ces pratiques concernent le paysage, en tant qu'environnement du sujet, milieu lié à un investissement et à un « faire » spécifique. Elles relèvent parfois de la performance (c'est le cas pour le « Laboratoire d'Art Urbain Stalker »). Elles impliquent toujours le corps d'un voyageur (qui peut être écrivain, photographe, artiste, etc.). Parfois le sujet itinérant est le visiteur lui-même, qui sort désormais de sa passivité. La contrainte est au fondement même de l' "articité » (les règles de la prosodie, de l'harmonie musicale ou encore de la perspective sont de fait contraignantes). Régler le déplacement par un protocole, c'est le démarquer d'une manière banale et automatique d'aller d'un endroit à un autre ; c'est lui conférer une artificialité et, pour ainsi dire une appartenance au champ de l'art. Toute espèce de déplacement contraint (quelle que soit la restitution sur laquelle il débouche) suggère que le voyage est en lui-même déjà une pratique qui relève peu ou prou de l'art ; elle suppose que la création ne commence pas avec la recomposition du vécu, mais avec le vécu lui-même. On comprend que les protocoles - qui sont susceptibles de contribuer à l'« artialisation » des conduites humaines - intéressent les artistes proches de la performance; elles attirent également les voyageurs qui souhaitent opérer une valorisation de l'expérience itinérante en elle-même.

Ces démarches - qui cultivent une certaine affinité avec la recherche expérimentale - sont souvent relayées par l'emploi d'appareils qui permettent l'enregistrement des apparences (la photographie, la vidéo...) ou par des modalités d'écriture que l'on pourrait dire «plates », constatives, méthodiques. L'utilisation d'appareils, technologiquement perfectionnés et considérés comme fiables, tend à conforter une posture de type empirique. Elle travaille à conférer à ces pratiques une allure de scientificité ; celle-ci paraît de fait souhaitée puisque les membres du collectif «Stalker » parlent de «Laboratoire d'Art Urbain », puisque Thierry Girard emploie l'expression d' observatoire du paysage $»^{1}$; Quant à Carol Dunlop et Julio Cortázar, prenant modèle sur les grands explorateurs, ils mentionnent précisément à chaque halte la température, l'orientation de leur véhicule, les conditions météorologiques... susceptibles de conditionner leurs observations.

1 C'est moi qui souligne. 
Ces entreprises trouvent en la photographie un outil de choix. L'image possède de fait une singulière aptitude à la « dé-familarisation ». Le sujet enregistré sur un cliché se trouve revêtu d'une certaine étrangeté, car l'image décolle, isole la vision des normes inconscientes qui commandent ordinairement notre appréhension des choses ${ }^{1}$. Cette aptitude de l'image n'est pas sans rejoindre certains effets induits par la situation du voyage, qui est susceptible de réactiver une attention au réel. Elle converge aussi avec les conséquences recherchées par la contrainte du déplacement.

Les démarches à contrainte partent du principe qu'un protocole établi au départ peut modifier la relation au territoire. Or la photographie est un dispositif avant d'être une représentation ; elle est une manière de se placer face à la réalité des lieux, qui est elle-même assortie d'un certain nombre de règles : elle fixe un moment très précis ; elle isole un pan de réel de son contexte, elle est tributaire d'une situation de l'opérateur dans l'espace, d'un angle de vue... Le choix d'un appareil conditionne certaines modalités d'accueil du réel, génère un régime donné de perception des apparences. Le mécanisme de l'outil détermine une certaine forme d'ouverture au monde (que le photographe ne peut pour partie pas modifier). Elle implique chez l'opérateur une part d'activité et une part de passivité. La prise de vue impose des règles, susceptibles de surprendre nos modes habituels de perception. Il n'est donc pas étonnant qu'elle rencontre la contrainte au sein de certaines démarches qui associent un écrivain et un photographe ou encore chez des praticiens qui travaillent selon des protocoles très précis (Thierry Girard, Pierre Devin, Bernard Plossu, Claude Pauquet...). On trouve aussi des écrivains, tel François Bon (Paysage fer), qui assortissent leur texte de continuels rapprochements avec la photographie.

Dispositif photographique et parcours contraint sont dès lors les instruments convergents d'un OUvroir de PAYsage POtentiel. Les démarches que j'ai envisagées répondent à des préoccupations qui se développent aujourd'hui en matière d'aménagement du territoire. Elles sont dotées d'une dimension politique. Elles travaillent à changer la façon commune de percevoir le paysage et sont, dans une certaine mesure, susceptibles d'informer la société.

1 J.-M. SCHAEFFER, L'image précaire. Du dispositif photographique, Paris, Seuil, « Poétique », 1987, p. 170. 
